\title{
The engineer and the clinician: Understanding the work output and troubleshooting of the HeartMate II rotary flow pump
}

\author{
W. Scott Arnold, MD, and Kevin Bourque, MSME
}

The HeartMate II (Thoratec, Pleasanton, Calif) left ventricular assist device has been implanted in more than 10,000 patients worldwide. It has been approved by the Food and Drug Administration for both destination and bridge-totransplant therapy. ${ }^{1-5}$ The design has changed from the original HeartMate XVE, a volume-displacement (VD) pump, to the current continuous, axial, and rotary pump. The pump has a high-speed, axially oriented, impeller with a helical vane geometry driven by a 3-phase, direct current electrical motor. Device flows are not measured directly but rather are inferred from pump speed and pump power consumption according to the known relationship among speed, power, and flow.

Clinicians are accustomed to thinking in terms of the human heart, a VD pump. The heart converts the chemical energy of adenosine triphosphate into mechanical energy at the level of the sarcomere. ${ }^{6-8}$ The muscle contracts, generating pressure work $($ work $=$ pressure $[\mathrm{P}] \times$ change in volume $[\mathrm{dV}] /$ change in time $[\mathrm{dT}])$. VD pumps generate pressure and thus flow in inverse proportion to the vascular resistance. As resistance increases, cardiac work increases.

In contrast, in a rotary pump (whether axial or centrifugal), as resistance increases, the work (and hence power [power $=$ work/time]) decreases. This seems paradoxical to a clinician who envisions the pump "pushing against resistance" and therefore, similar to a VD pump, doing more work. This concept is incorrect, but to an engineer, the fact that decreased flow means less work is intuitive.

The trouble-shooting algorithms for the HeartMate II are determined from an analysis of pump power (consumption) and speed. Therefore, a more intuitive understanding of rotary flow pump dynamics is needed. We will attempt to clarify the differences between VD and rotary flow (RF) pumps with respect to fluid dynamics and work.

VD pumps are particularly good at generating pressure against resistance. Examples include the Abiomed 5000 left ventricular assist device, the original HeartMate XVE (which generated the data for the Randomized Evaluation

\footnotetext{
From the Department of Cardiothoracic Surgery, Carilion Clinic, Roanoke, Va.

Disclosures: Kevin Bouque is employed by Thoratec, the maker of HeartMate. The other author has nothing to disclose with regard to commercial support.

Received for publication March 15, 2012; revisions received July 3, 2012; accepted for publication July 26, 2012; available ahead of print Nov 7, 2012.

Address for reprints: W. Scott Arnold, MD, Department of Cardiothoracic Surgery,

Carilion Clinic, 2001 Crystal Spring Ave, Suite 201, Roanoke, VA 24014

(E-mail: wsarnold@ carilionclinic.org).

J Thorac Cardiovasc Surg 2013;145:32-6

$0022-5223 / \$ 36.00$

Copyright $(2) 2013$ by The American Association for Thoracic Surgery

http://dx.doi.org/10.1016/j.jtcvs.2012.07.113
}

of Mechanical Assistance for the Treatment of Congestive Heart Failure [REMATCH] trial $^{9}$ ), and the human heart. They can, at the expense of increased work and energy consumption, keep flows constant against increasing resistance, thereby generating greater pressures. Hydraulic work in this system is most simply related to pressure and volume $(\mathrm{W}=$ $\mathrm{P} \times \mathrm{dV} / \mathrm{dT}$ ). During the cardiac cycle, as illustrated in the pressure-volume loop (Figure 1), volume increases during diastole, with a modest increase in pressure dependent on compliance (Figure 1, point A). The energy for this filling is the residual kinetic energy from the previous systole minus work done over distance and against elasticity and friction. The heart fills passively, with the exception of the external suction forces transmitted from the expanding thoracic cavity during inspiration. The more interesting phase is isovolumic contraction (Figure 1, point B). Here, no hydraulic work is done. No ejection occurs and thus the blood travels no distance and because work $=$ force $\times$ distance, no hydraulic work occurs. The contraction is isovolumic, and, therefore, there is no $\mathrm{dV} / \mathrm{dT}$ and, thus, no true pressure work. However, this is the phase of maximal oxygen consumption by the myocytes. ${ }^{6}$ Is this a paradox? Not in terms of overall thermodynamics. Just as an isometric skeletal muscle contraction does no mechanical work (force $\times$ distance) yet still exerts force, the isovolumic contraction generates pressure and potential energy; however, work is not done until the aortic valve opens and cardiac ejection takes place (Figure 1, point C). During the isovolumic contraction, adenosine triphosphate is split, and its chemical energy is converted into mechanical energy by the myosin heads. The sarcomeres contract generating pressure. Chemical energy becomes potential energy stored as pressure. With ejection, the pressure results in contraction (a change in volume, $\mathrm{dV} / \mathrm{dT}$ ) and, thus, work as forward flow.

VD pumps are well-suited for maintaining constant flows against varying afterload. However, at very high resistances or flow stop (eg, an aortic crossclamp), the pump fails in short order. Mechanical VD pump systems fail mechanically, or the electrical draw exceeds the circuit ampacity. The heart, as a modified VD pump, is, of course, more complex. It generates variable output volumes according to preload, afterload, and contractility. The failure of the intact heart at high afterload gives us the apparent "descending limb" of the Frank-Starling curve, the mechanism of which is complex but is due primarily to progressive endocardial ischemia (rather than the sarcomere-slip theory implied by isolated muscle strip preparations). 

Abbreviations and Acronyms
$\mathrm{dT}=$ change in time
$\mathrm{dV}=$ change in volume
$\mathrm{P}=$ pressure
PI $=$ pulsatility index
$\mathrm{RF}=$ rotary flow
$\mathrm{VD}=$ volume-displacement

In contrast, an RF pump, whether axial or centrifugal, generates flow, with the amount of pressure dependent on resistance to flow. This might seem like a subtle distinction, but it is the basis of the apparent paradoxical relationship between resistance and work.

For example, if we clamp the outflow graft (Figure 2) of the HeartMate II, the work done by the motor turning the impeller decreases. To a clinician who is used to cardiac oxygen demand and wall stress increasing dramatically with application of an aortic crossclamp, this seems paradoxical. Although the impeller might seem to be "pushing against an infinite resistance," the true electrical and mechanical effort of the pump actually decreases. Why?

RF pumps consist of a motor that turns an impeller that rotates at high speed inside its housing. The impeller accelerates the fluid either forward (in the case of axial flow pumps) or outwardly (in the case of centrifugal pumps). The actual physics of impeller function is surprisingly complex. The impeller need not be a cambered, aerofoil shape (like a wing), although some are. Its ability to generate flow is dictated by rotational speed, blade radius, blade pitch, and blade height, as described by Euler's pump theory. ${ }^{10}$ Fluid dynamics are determined by the expansions of Newton's second law (force $=$ mass $\times$ acceleration) and the first law of thermodynamics, conservation of energy. We are most familiar with these relations as expressed in Bernoulli's equation. The total fluid energy at point A is the total of pressure energy plus the kinetic energy of motion $\left(1 / 2 \mathrm{mv}^{2}\right)$ plus the potential energy of gravity (pgh). This total energy is conserved at point $\mathrm{B}$. Thus, $\mathrm{P}_{\mathrm{a}}$ $+1 / 2 \mathrm{mv}_{\mathrm{a}}^{2}+p \mathrm{gh}_{\mathrm{a}}=\mathrm{P}_{\mathrm{b}}+1 / 2 \mathrm{mv}_{\mathrm{b}}^{2}+p \mathrm{gh}_{\mathrm{b}}$.

Bernoulli's equation can be derived by several methods. It can be derived from Newton's second law (force = mass $\times$ acceleration), from the conservation of energy (work $=$ force $\times$ distance), or by distilling much more complex equations such as Euler's equation (not to be confused with Euler's pump theory) and the Navier-Stokes equation. The latter 2 equations are complex, compound, partial differential equations that start with 3-dimensional vector depictions of finite elements of flow. Interestingly, all relate conservation of momentum to fluid systems and can be simplified roughly to Bernoulli's equation.

Simply, as the impeller rotates, it accelerates the fluid dramatically, adding its mechanical energy to the " $1 / 2 \mathrm{mv}^{2 "}$ " $\mathrm{ki}$ netic energy component as flow. At the exit, the fluid slows, shifting the added energy of flow back to "P," pressure. The amount of this shift back depends on outflow resistance. At the inlet, suction is created. In fact, enough suction can be generated to induce cavitation, which is the bubbling that occurs when the pressure decreases to less than the vapor pressure of the fluid. In addition to the power consumed by the pump to produce hydraulic work, power is consumed just to overcome viscous friction, resistance in electrical conductors, and the like. Such losses are usually regarded as "overhead" and are largely independent of flow. Although not necessarily constant, within the typical operating range, this friction component generally does not interfere with the direct relationship between flow and power.

When an outflow obstruction is present in a RF system (increased afterload), flow decreases and less work is done (again, work $=$ force $\times$ distance). The rotor spins at the same speed, but the impellers contact and "thrust" less fluid and, thus, do less work. The energy demand decreases. Clamping the outflow graft of the HeartMate II leaves the impeller rotating in a swirling, but axially static, volume of fluid. At this point of no flow, total work decreases dramatically, and power consumption is entirely due to overcoming friction.
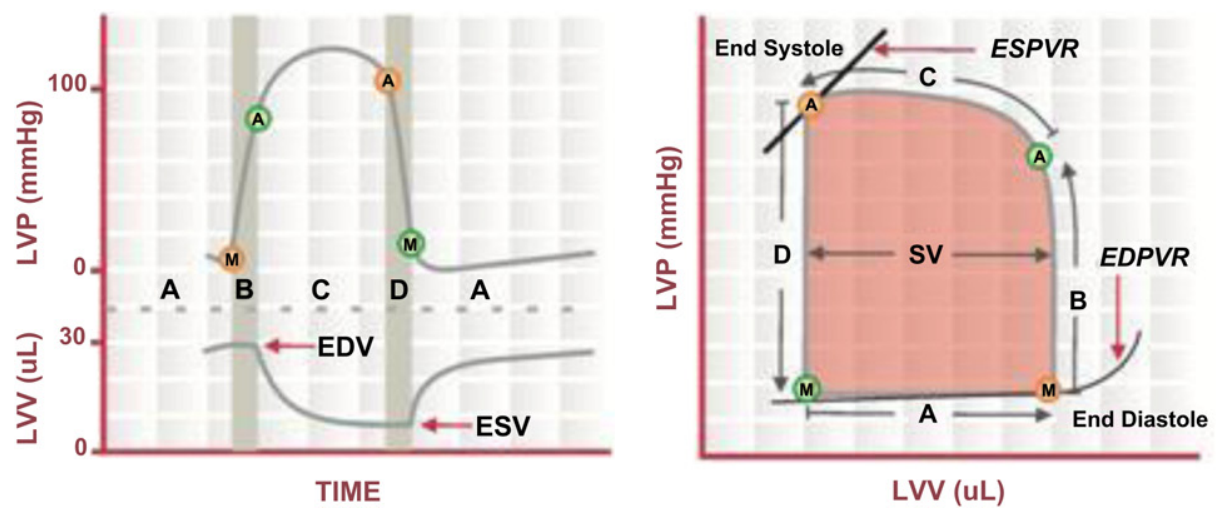

\section{A. Diastolic Filling \\ Mitral Valve Closes}

B. Isovolumic Contraction

A Aortic Valve Opens

C. Ejection

A Aortic Valve Closes

D. Isovolumic Relaxation

Mitral Valve Opens

FIGURE 1. Cardiac pressure-volume loop. EDPVR, End-diastolic pressure-volume relationship; $E D V$, end-diastolic volume; $E S P V R$, end-systolic pressure-volume relationship; $E S V$, end-systolic volume; $L V P$, left ventricular pressure; $L V V$, left ventricular volume; $S V$, stroke volume. 


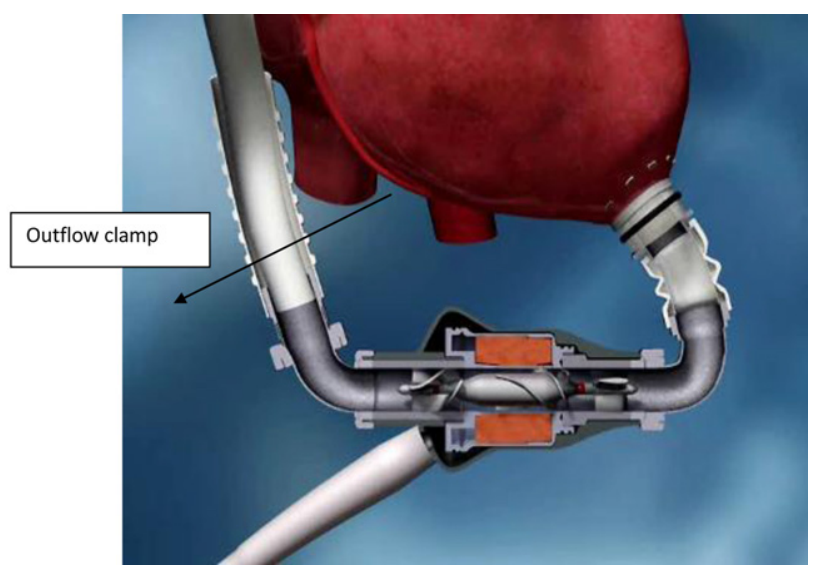

FIGURE 2. Outflow clamp crossclamped.

In contrast to a VD pump, a RF pump handles low/noflow situations well, with a decrease in work, rather than an increase. This is a fundamental difference.

This effect can be readily observed by examining an ex vivo HeartMate II test loop and occluding flow. One can also confirm this generalized behavior of an RF pump with other types of centrifugal machines. For example, we examined a domestic pool system run by a variable-speed, RF pool pump equipped with an in-line Watt-meter (IntelliFlo; Pentair, Minneapolis, Minn) and varied the inflow and outflow resistances and observed power consumption. The results are shown in Figure 3.
One helpful analogy to a RF pump taken to a no-flow state is that of an automotive flywheel disengaging from a clutch. It still spins, but the rotational energy is not transferred to the drive train. Engine effort is less. Another is the aerofoil rotor of a helicopter, which generates lift according to the Bernoulli principle, similar to an airplane wing. It moves through air molecules, generating lift according to rotor speed, rotor pitch, and air density. At high altitudes, the air density is less. Fewer air molecules are available to accelerate, and, thus, lift decreases. Taken to a theoretical extreme, the rotor would spin in a vacuum and do no lift work, and the engine would feel little strain.

When the HeartMate II "disengages" (ie, flow stop), its function becomes more analogous to a mixer than a pump. The impeller can contact and accelerate only that volume of fluid within the housing and can thus do work only on that volume of fluid.

With this in mind, we can examine the HeartMate II in more depth. During cardiac systole, RF pump work increases. Again, this might seem counterintuitive. The pump is being "assisted" by cardiac contraction. In fact, the flow across the pump does increase. The capacity of the pump to accelerate fluid is in excess of the physiologically supplied volumes, and it can easily "engage" or contact and accelerate more fluid. The clutch engages, so to speak, and more work (and more power requirement) is done. This is the basis of the HeartMate II pulsatility index (PI), as defined in the user's guide (Figure 4). ${ }^{11}$ The pump "knows" only revolutions per minute and the power

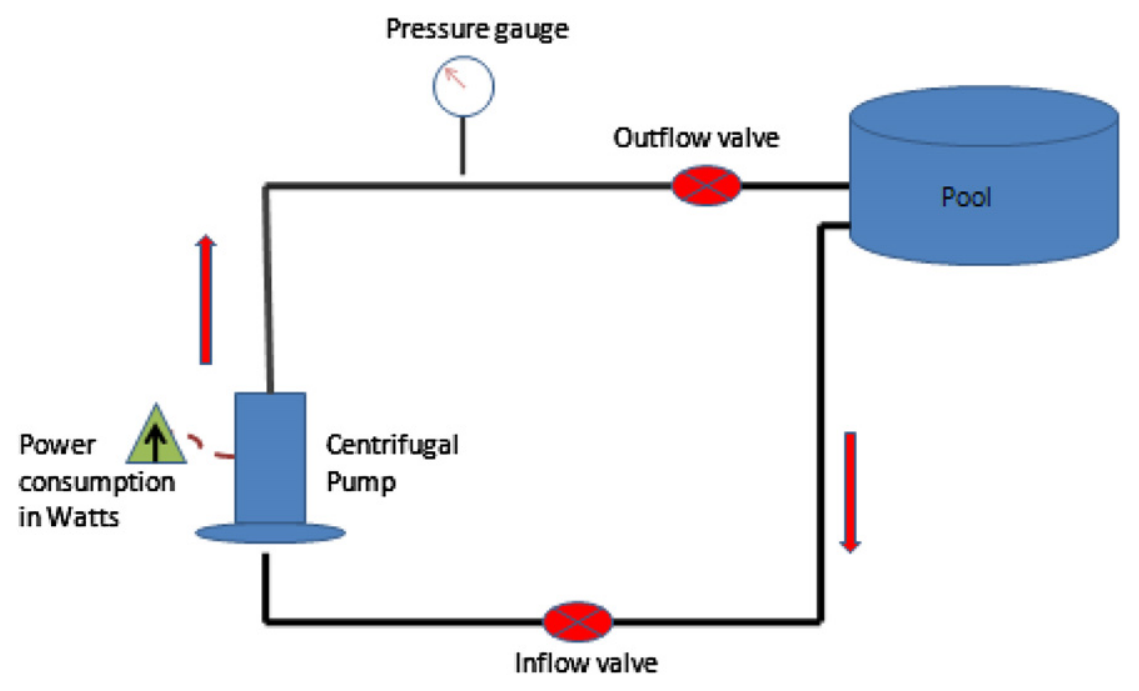

\begin{tabular}{|l|l|l|l|l|}
\hline RPM's & $\begin{array}{l}\text { Pressure } \\
\text { (PSI) }\end{array}$ & $\begin{array}{l}\text { Inflow } \\
\text { valve }\end{array}$ & $\begin{array}{l}\text { Outflow } \\
\text { valve }\end{array}$ & $\begin{array}{l}\text { Watts } \\
\text { used by } \\
\text { pump }\end{array}$ \\
\hline 2350 & 12 & open & open & 780 \\
\hline 2350 & 0 & closed & open & 450 \\
\hline 2350 & 18 & open & closed & 463 \\
\hline
\end{tabular}

FIGURE 3. Centrifugal pump circuit diagram for Pentair Intellifo variable speed pump, with continuous power consumption displayed in Watts. 
- $\mathrm{PI}$ is a measure of the amount of pulsatility seen by the pump over a fifteen-second interval. This value relates to the amount of native heart function.

$$
P I=\frac{(\text { Max Flow })-(\text { Min Flow })}{\text { Average Flow }}
$$

- $\mathrm{PI}$ is calculated from pump power normalized by mean power

FIGURE 4. Derivation of pulsatility index $(P I)$.

required to maintain that speed. There is no true flow detector. With native cardiac ejection, as noted, power usage increases, and, thus, the implied flow is greater. The lower the PI, the greater the amount of support provided by the pump. The greater the PI, the more the native heart is ejecting. The PI will therefore vary with the state of hydration, contractility, right ventricular function, and exercise.

Troubleshooting now becomes a bit more intuitive (Figures 5 and 6). If the controller shows decreased power usage with a stable rotor speed, this implies decreased work and, thus, decreased flow. This could result from decreased inflow due to volume depletion or cannula malposition or to increased afterload resulting from vasoconstriction, hypertension, or cannula geometry. Poor inflow owing to volume depletion versus poor drainage can be differentiated with echocardiography.

Increased power consumption with increased pulsatility index suggests more "assisted flow" and, thus, native cardiac recovery or volume repletion from a dehydrated state.

Thrombus can confound the reported flow estimate by changing the assumed invariant relationship among speed, power, and flow. ${ }^{12}$ Thrombus can cause either increased power consumption if the predominant burden is on the bearings, requiring more friction work (leading to a spurious indication that flow has increased), or decreased power consumption if the predominant physiology is decreased flow owing to clot in the housing inflow or outflow. Perhaps the most vexing condition is when both effects approximately balance, and power remains roughly the same, with an occult reduction in flow and clinically poor perfusion, with evidence of poor ventricular drainage on echocardiography.

Rotary pumps are ideally suited for high-flow work with variable resistance. They are simple and durable but are fundamentally different in terms of hydrodynamics from VD pumps. We hope this discussion has been s helpful in making the understanding of the HeartMate II more intuitive.

\section{References}

1. Bull DA, Reid BB, Selzman CH, Mesley R, Drakos S, Clayson S, et al. The impact of bridge-to-transplant ventricular assist device support on survival after cardiac transplantation. $J$ Thorac Cardiovasc Surg. 2010;140:169-73.

2. John R, Pagani FD, Naka Y, Boyle A, Conte JV, Russell SD, et al. Post-cardiac transplant survival after support with a continuous-flow left ventricular assist device: impact of duration of left ventricular assist device support and other variables. J Thoracic Cardiovasc Surg. 2010;140:174-81

3. Miller LW, Paganini FD, Russell SD, John R, Boyle AJ, Aaronson KD, et al. Use of a continuous-flow device in patients awaiting heart transplantation. $N$ Engl J Med. 2007;357:885-96.

4. Paganini FD, Miller LW, Russell SD, Aaronson KD, John R, Boyle AJ, et al. Extended mechanical support with a continuous-flow rotary left ventricular assist device. J Am Coll Cardiol. 2009;54:312-21.

5. Slaughter MS, Rogers JG, Milano CA, Russell SD, Conte JV, Feldman D, et al Advanced heart failure treated with a continuous-flow left ventricular assist device. $N$ Engl J Med. 2009;361:2241-51.

6. Suga H. Ventricular energetics. Physiol Rev. 1990;70:247-77.

7. Goto Y, Slinker BK, LeWinter MM. Decreased contractile efficiency and increased nonmechanical energy cost in hyperthyroid rabbit heart: relation between $\mathrm{O}_{2}$ consumption and systolic pressure-volume area or force-time integral. Circ Res. 1990;66:999-1011.

8. Kass DA, Maughan WL. From "Emax" to pressure-volume relations: a broader view. Circulation. 1998;77:1203-12.

9. Rose EA, Gellins AC, Moskowitz AJ, Heitian DF, Stevenson LW, Dembitsky W, et al. Long-term use of a left ventricular assist device for end-stage heart failure. N Engl J Med. 2001;345:1435-43.

10. Grundfos Research and Technology. The centrifugal pump. Available at: http:// dk.grundfos.com/content/dam/Global\%20Site/Industries $\% 20 \% 26 \% 20$ solutions/Industry/pdf/The_Centrifugal_Pump.pdf. Accessed June 23, 2012.

11. Thoratec Corporation. Heartmate II LVAS troubleshooting guide; revision C, document number 1002083. Pleasanton, CA: Thoratec Corporation; 2010 .

12. Bhamidipati CM, Ailawadi G, Bergin J, Kern JA. Early thrombus in a Heartmate II left ventricular assist device: a potential cause of hemolysis and diagnostic dilemma. J Thorac Cardiovasc Surg. 2010;140:e7-8.

\begin{tabular}{|c|c|}
\hline Backflow & $\begin{array}{l}\text { - Low flow or low speed alarms, dilated ventricle } \\
\text { - Low speeds with hypertensive patient }(B P>120)\end{array}$ \\
\hline Recovery & $\begin{array}{l}\text { - Increased PI } \\
\text { - Aortic valve opening on every beat } \\
\text { - Increased myocardial oxygen consumption }\end{array}$ \\
\hline Exercise & $\begin{array}{l}\text { Increased PI due to additional venous return } \\
\text { - Aortic valve opening }\end{array}$ \\
\hline Pump Thrombus & $\begin{array}{l}\text { - Increase in power (usually gradual over hours or days) } \\
\text { - Decrease in PI } \\
\text { - Inability to unload LV with increasing speed } \\
\text { Inability for pump to maintain speed }\end{array}$ \\
\hline Flow Path Obstruction & $\begin{array}{l}\text { - Decrease in flow and flow estimate } \\
\text { - Decrease in power } \\
\text { - Inability to unload LV with increasing speed }\end{array}$ \\
\hline
\end{tabular}

FIGURE 5. Clinical scenarios and associated device/hemodynamic changes. $B P$, Blood pressure; $P I$, pulsatility index; $L V$, left ventricle. 


\begin{tabular}{|c|c|c|c|}
\hline \multicolumn{2}{|c|}{$\begin{array}{c}\text { What significant changes am I seeing? } \\
\text { (Displayed Flow) }\end{array}$} & $\begin{array}{l}\text { Pulsatility Index } \\
\text { (PI) }\end{array}$ & Pump Speed
\end{tabular}

FIGURE 6. Heartmate II (Thoratec, Pleasanton, Calif) troubleshooting chart. $L V$, Left ventricular. 